\title{
$X$-ray powder diffraction structural studies of lithol red pigments
}

Wieslaw Lasocha ${ }^{1}$, Marta Grzesiak-Nowak ${ }^{1}$, Alicja Rafalska-Lasocha ${ }^{1}$, Marcin Oszajca ${ }^{1}$, Piotr Goszczycki ${ }^{1}$, Katarzyna Ostrowska $^{1}$, Joanna Kuncewicz ${ }^{1}$, Wojciech Macyk ${ }^{1}$

${ }^{1}$ Faculty Of Chemistry Jagiellonian University, Krakow, Poland

E-mail: lasocha@chemia.uj.edu.pl

Lithol reds belong to the group of azo pigments which were popular artists' pigments at the beginning of the twentieth century. Under the name lithol red pigment, one can find a family of sodium (PR 49), barium (PR 49:1), calcium (PR 49:2), and strontium (PR 49:3) salts of diazotised Tobias (2-naphthylamine-1-sulphonic) acid coupled with 2-naphthol. The colour of the pigment ranges from yellowish red (sodium salt) to bluish red (strontium salt), depending on the metal cation. The main drawback of lithol red is its very poor lightfastness, which has profound implications for its artistic use (e.g. Mark Rothko's Seagram and Harvard Murals) [1].

The mechanism of deterioration and fading of lithol reds is until now unknown. Some literature data suggest that this pigment is fading under the influence of light and the binding medium. Fading of a pigment can be also connected with the changes in its crystal structure, so the aim of the presented research was the study of the crystal structures of the lithol salts with sodium, barium and ammonium cations.

Sodium salt is the starting compound for production of the other lithol salts by means of a ion-exchange process whereby $\mathrm{Ba}$ or $\mathrm{NH} 4$ ions replace the $\mathrm{Na}$ ions. Due to this process is inefficient and some Na salt almost always remains, it is very difficult to obtain a pure lithol salt suitable for crystallographic studies. Recently we have undertaken efforts to obtain, purify and recrystallize a few lithol salts. As a result we have obtained pure lithol salts suitable for powder diffraction studies and solved the crystal structures of sodium and ammonium salts. In the case of barium salt we were only able to determine its lattice parameters and to find the most probable space group.

For powder diffraction experiments the samples were placed into glass capillaries and all XRPD measurements were performed using laboratory X-ray source (an X'PERT PRO MPD apparatus, CuKa1 radiation $(\lambda=1.54059 \AA$ ), equipped with diffracted-beam graphite monochromator, focusing mirror and a PIXcel PSD detector). Crystal structures were solved by global optimization techniques [2] and refined by restrained Rietveld method [3].

The investigated pigment samples were also subjected to artificial aging process using a $150 \mathrm{~W}$ Xenon lamp. After the light exposure all samples were examined by means of: UV-Visible (UV-Vis) Absorption, Fourier-Transformed Raman spectroscopy (FT Raman), Fourier-Transformed micro-Infrared spectroscopy (FT-MIR) in the Attenuated Total Reflection (ATR) mode and XRPD techniques.

Obtained results will be presented and discussed during the conference.

Figure 1. Crystal structures of: a/ sodium lithol salt b/ ammonium lithol salt. Colour assignments: grey - C, blue - N, red $\mathrm{O}$, yellow - S, violet - $\mathrm{Na}$, small circles $-\mathrm{H}$.

[1] Stenger, J. et al. (2010) e-PS. 7, 147-157.

[2] Favre-Nicolin. V. et al. (2002) J. Appl. Cryst., 35, 734-743.

[3] Petricek. V. et al. (2014) Z. Kristallogr. 229, 345-52.
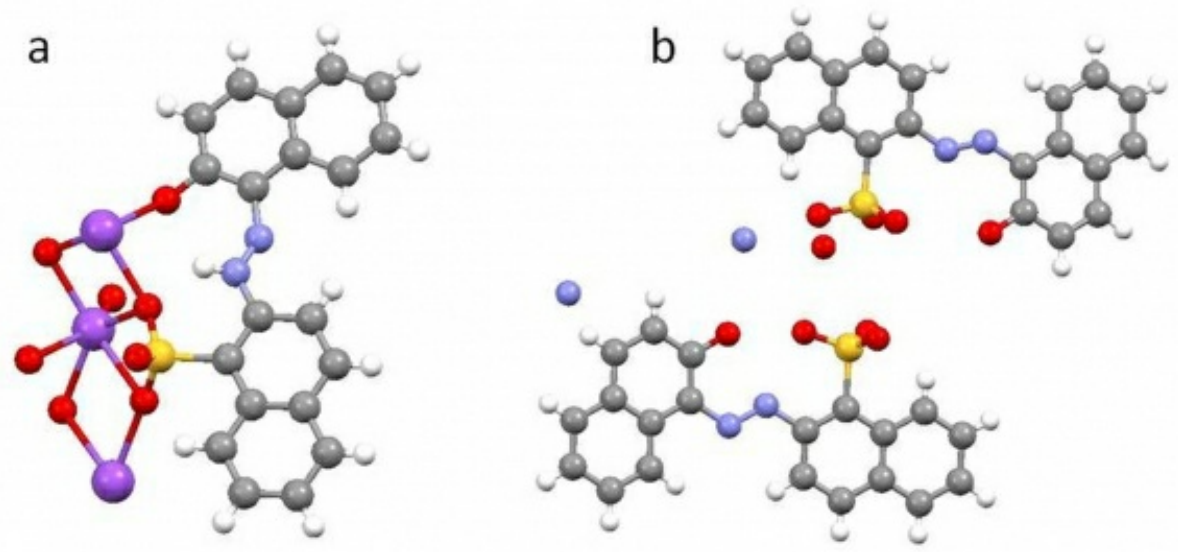

Keywords: lithol red pigments, $\mathrm{x}$-ray powder diffraction, structural studies

Acta Cryst. (2017). A73, C1400 